\title{
Interdisciplinary collaborations at work in brain-machine interfacing
}

\author{
Jonathan Brumberg, Assistant Professor, Speech-Language-Hearing, \\ University of Kansas
}

$\mathrm{P}$ ublic universities are facing growing uncertainty, specifically in our ability to recruit students and secure appropriate funding to support our research programs. In this paper, interdisciplinary and translational research are discussed as avenues of growth that have the potential to increase research productivity, and diversify institutional research portfolios capable of weathering these unstable times. Cognitive neuroscience, neurocomputational modeling and brain-machine interface research will be discussed as frameworks for pursuing interdisciplinary and translational research.

Brain machine interfacing (BMI) is a relatively recent addition to the field of neuroscience, and derives from a longstanding history of cognitive neuroscience. It is truly an area of applied neuroscience in the sense that the intended goals of BMIs are to use neurological activity for interacting with computerbased devices without direct manipulation by a human controller (cf. keyboard typing, mouse clicking, etc.). Such an achievement depends greatly on our ability to quantitatively model the brain (i.e., neurocomputational modeling), and associate those measurements with sensory perception and motor behavior. One major application of BMI devices is for rehabilitation and assistive technology, including the remote control of movement devices (robotic exoskeletons, wheelchairs) and communication systems (e.g., augmentative and alternative communication devices). The development, deployment and use of BMI technology relies on a coordinated effort to effectively integrate expertise from a number of disciplines according to the needs underlying the specific BMI application.

For success in an academic research environment, much of this expertise can be drawn, or developed, from student involvement, particularly at the graduate level. Management of such an interdisciplinary project, however, requires team leadership with sufficient experience in many, if not all, areas of expertise. The present paper begins with an overview of cognitive and computational neuroscience research in speech and language, follows with examples of BMI research for communication and concludes with a discussion of strategies for facilitating interdisciplinary research, recruiting future generations of researchers, and engaging our regional and national communities. Speech, language and hearing neuroscience is specifically explored as a prime example of interdisciplinary research with many potential translational appli- 
cations. Three neurotechnological applications are discussed, each of which have important societal value: cochlear implants to restore hearing for those with profound hearing loss, deep brain stimulators for ameliorating motor symptoms of Parkinson's disease, and brain-machine interfaces to enable communication for individuals with locked-in syndrome. Advancement in areas such as these have the potential to drive even greater discoveries and benefit to society. Specific suggestions are provided for fostering interdisciplinary research at public universities.

\section{Introduction}

Cognitive and computational neuroscience has for many years been at the forefront of discovering the link between brain and behavior as we experience the world. Using advanced neuroimaging and electrophysiological tools such as functional magnetic resonance imaging (fMRI), positron emission tomography (PET) and electroencephalography (EEG), we have been able to infer relationships between changes in the metabolic and electrical activities in the brain to distinct sensory, motor and cognitive functions. Areas of particular interest are in the neuroscience of reading, speech and language production, speech perception and language comprehension. It is now possible to translate the experimental conclusions of cognitive and computational neuroscience into practice for diagnosing and rehabilitating disorders with a neurological deficit. The remainder of this paper will discuss the interdisciplinary basis of speech and language neurological research, translational applications of neuroscience research and end with suggestions on supporting the growth of interdisciplinary research, in an otherwise uncertain time, using applied speech neuroscience as a framework.

Cognitive \& computational neuroscience in speech-language research

Speech and language are uniquely human capabilities that in many ways define our species, yet are also some of our most underrated, or overlooked abilities. Often, it is not until a deficit is encountered that we truly realize the importance of speech and language in our daily lives. Deficits in speech, language and hearing often have a neurological origin, which requires comprehensive study of the related brain structures and activations in individuals with, and without disorder. Some of the earliest cognitive neuroscience investigations took place in the nineteenth and early twentieth centuries as researchers were uncovering the critical role of specific areas of the brain for producing speech (e.g., Broca's area) and understanding speech (e.g., Wernicke's area). One of the most frequent methods used at the time relied on associating post-mortem anatomical examinations of human brains with the presence or absence of specific production or comprehension deficits. At the same time, investigators were also discovering that electrical stimulation of brain tissue could elicit behavioral responses and that specific deficits or overexcitation led to the occurrence of seizures, which could be alleviated by resection of the dysfunctional tissue. Importantly, both approaches provided the first steps toward understanding how the brain mediates speech and language, and whether they realized it or not, these early cognitive neuroscientists 
were engaging in interdisciplinary research involving medical treatment of patients, neuroscientific study and an understanding of speech-language processing. This last point is very important for the topic of the present paper; cognitive neuroscience has been an interdisciplinary field from its inception.

In the twentieth century, the advent of non-invasive functional neuroimaging methods (PET, fMRI) helped to expand speech and language neuroscience beyond the study of disorder using innovative techniques. With these new methods, it was finally possible to systematically investigate, in large populations of individuals without impairment, prior conclusions regarding the speech and language processes of the brain obtained from anatomical and electrical stimulation studies [see Price (2012); and Indefrey \& Levelt (2004); for reviews]. In the years since, multiple regions have been shown to contribute to speech preparation and production, as opposed to a single region described by Broca. Similarly, speech perception and language comprehension are subserved by a number of anatomical regions working in concert. In more recent years, the dramatic explosion of computational power has led to the development of neurocomputational models of speech and language processes (Saltzman \& Munhall 1989; Guenther et al. 2006; Houde \& Nagarajan 2011). These models extended previous theoretical models by using computer programs to simulate the information processing by the brain according to empirical evidence from functional neuroimaging studies. As a result of the advances in modern cognitive and computational neuroscience, the study of the brain mechanisms of speech, language and hearing has grown even more interdisciplinary to include computer scientists, electrical \& biomedical engineers, linguists and psychologists (among many others). An engineering branch of computational neuroscience seeks to move from using a computer to model the brain toward the brain modeling, or controlling, a computer. This inversion of computational neuroscience is at the heart of brain-machine interfacing.

\section{Applied neuroscience}

The term applied neuroscience is used here to refer to any aspect of neuroscientific research that results in our ability to alter or influence neurological processes for rehabilitation. Some visible examples of this type of translational research include the neuropharmaceutical treatments (e.g., antidepressants) and neurologically targeted rehabilitation of stroke and traumatic brain injury. Some neurotechnological translational research examples include intervention in cases of sensorineural hearing loss via cochlear implants, and motor deficits associated with Parkinson's disease via deep brain stimulation. In both of these cases, neurological models were first constructed based on empirical evidence, then "inverted" to allow communication between computational devices and neurological tissue.

For developing cochlear implants, the physiology of the cochlea was first mapped and discovered to encode acoustic frequency according to location along the basilar membrane. This frequency information is then electrically transmitted through the auditory nerve to the nervous system via movement of the inner hair cells of the cochlea. For individuals 
with profound sensorineural hearing loss, encoded sounds are not transmitted to the central nervous system, though the location-frequency representations of the cochlea and auditory nerve are preserved. In accordance with this model of cochlear function, the cochlear implant uses a miniature computer to acquire and analyze incoming sound for its frequency content and electrically stimulate the cochlea at the appropriate location via implanted electrodes. In this way, the peripheral nervous system and a computational device are in direct communication to provide the central nervous system with restored hearing information for processing.

Similarly, in the case of deep brain stimulating implants, neuroscientfic investigations first uncovered the function and structure of the brain regions implicated in the motor deficits associated with Parkinson's disease. These structures, known as the basal ganglia, are important for selecting and initiating voluntary movements such as reaching with the arm or speaking. Two specific areas, the globus pallidus (GP) and subthalamic nucleus (STN), appear to be particularly affected in Parkinson's disease; their dysfunction causes the basal ganglia network, through its connection with neurological motor pathways, to excessively inhibit movement. Here, the network of structures are still intact, but are improperly activated resulting in the motor symptoms of Parkinson's disease. The deep brain stimulator addresses this neurological deficit through direct electrical stimulation of the GP and STN resulting in the disinhibition of voluntary motor behavior, and the alleviation of Parkin- sonian motor symptoms. Through a theoretical and quantitative description of this complicated neural circuit, it was possible to identify a specific portion of basal ganglia that under electrical stimulation had the potential to ameliorate the motor dysfunction of Parkinson's disease.

Both of the above applications, cochlear implants and deep brain stimulators, are great examples of the translational research potential at the intersection of engineering and neuroscience. In these examples, computers are interfacing directly with neural tissue in a one-way, input fashion (e.g., they direct the nervous system to act in a prescribed manner). The reverse design pattern in which neural activity controls a computer is also relevant for rehabilitation purposes, and are more traditionally considered brain-machine interfaces.

\section{Brain-machine interfacing}

In its most general sense, a brain-machine interface is any device designed to enable control of computational devices (e.g., robots, assistive or augmenting technology, communication aids) using brain activity alone, without any overt motor intervention. One area of particular interest is in the development of a BMI as an assistive communication device for individuals with quadriplegia and mutism, which is often the result of amyotrophic lateral sclerosis (ALS) or brainstem stroke. Both of these conditions can lead to locked-in syndrome (LIS; Plum \& Posner 1972), or the state in which an individual is completely unable to perform voluntary motor behaviors despite intact cognition and sensation. As a result of such profound speech and motor deficits, individuals with LIS are typically unable 
to use even the most advanced computerbased augmentative and alternative communication (AAC) devices. High-tech AAC devices are currently capable of detecting the smallest amount of voluntary movement behavior (e.g., muscular activity and eye-gaze location) to facilitate artificially aided communication, but even these capabilities are ineffective for individuals with LIS. In contrast, brain-machine interfaces are an ideal alternative for individuals with LIS; a BMI requires only a consistent and reliable source of neurological activity for mapping user intentions onto a communication device.

Principles of BMI development. A number of varieties of BMI devices for communication have been developed in recent years, each focusing on some aspect of neurological activity that can be willfully modulated by a participating user. Some examples include the P300 Speller (Donchin et al. 2000), sensorimotor rhythm keyboard spellers (Miner et al. 1998), event-related desynchronization keyboards (Neuper et al. 2003; Obermaier et al. 2003) and steady state visually evoked potential spellers (Friman et al. 2007). See (Brumberg \& Guenther 2010) for a review of each of these techniques. Each BMI example follows a core set of design principles in which a number of factors are optimized. These factors include:

1. Choosing the desired outcome

2. Determining the outcome delivery method

3. Selection of information bearing neural signals from participant

4. Statistical signal processing: mapping brain signals to outcomes

5. Training / treatment
All of the previously mentioned BMI examples selected communication as the desired outcome, and typing for the delivery method. Each example differed in the type of neurological activity used for the BMI, ranging from neural signals related to intended motor actions (e.g., imagined or attempted limb movements or visual evoked potentials), but all used non-invasive techniques involving the recording of electroencephalography. The statistical mapping procedures often depend on the neurological signal acquired for controlling the device, but usually rely on some form of machine learning algorithm. An alternative BMI device to those mentioned enables continuous control of an artificial speech synthesizer, with the long-term goal of providing a means for fluent speech production (Brumberg et al. 2010). This method also selects communication as the desired outcome, but uses continuous synthesizer control with instantaneous auditory output as the delivery method. For this BMI, motor-related neurological signals have been targeted both from intracortical microelectrodes (Brumberg et al. 2010) and electroencephalography. The last principle, training / treatment, is an area of recent attention and can most benefit from collaboration with speech-language pathologists specializing in AAC in order to help BMI users learn the skills needed to control their devices.

\section{Institutional support for interdisci- plinary research \\ Computational neuroscience, brain machine interface, and many initiatives for translational research result in out- comes that are both important to scien- tific advancement and have benefit to so- ciety. Research universities that support}


such initiatives can leverage these positive outcomes to further grow their local, regional and national standing. The question of how to best grow and support these lines of scientific inquiry is doubly important as public universities change their plans for future research in response to uncertainty in public higher education. Much of the discussion of uncertainty is related to the stability of federal funding of scientific research, and state funding of public education institutions. In many ways, as research faculty of public universities, we are being asked to do more with potentially much less. That said, public universities are in a unique position to thrive even in this difficult environment given our reputations as the face of our respective states' generators of educated citizens and engines of innovations. We also have a special ability to reach a broad cross-section of the population, ranging from our own students and faculty, to the local communities who represent either future students, or future voters who will be responsible for our long-term success. A focus on interdisciplinary and translational research is one avenue for of growth that is already fully compatible with the research missions of public universities. Further, these research efforts have the potential to truly do more with less (though we should always strive to do more with more), and the linking of multiple disciplines can spur new ideas to attract currently nontraditional contributors to public university research.

Engaging in community partnerships, and encouraging student participation in research.
The best way to increase the likelihood of continued success as a public institution is to give current and future voters a reason to support our endeavors. Many universities already participate in research experience programs where undergraduate students work alongside faculty and graduate student researchers in a laboratory setting. These experiences are critical for shaping future careers and perspectives, and should not be limited to undergraduates; opportunities for high school and middle school students can have a similar impact, and help direct younger students to our programs.

In the applied neurosciences, community and student engagement are especially important. Neuroscience education occurs primarily at the graduate level, and public perceptions of neuroscience often conjure images of medical doctors wearing white lab coats in hospitals. However, modern applied neuroscience in the form of brain machine interfacing is much more accessible -- commercial devices exist for acquiring neurological data (e.g., Emotiv Epoc), recent national initiatives in computer programming education has increased the number of young students with the skills to develop advanced software, mobile and educational computer hardware is more affordable than ever (e.g., RaspberryPi, Arduino), and a generation of "makers" are creating sophisticated robotics, electronics and even communication aids in their parents' basements. In addition, there is a growing awareness of speech-languagehearing disorders, their neural bases and technological remedies, and an increasing number of students interested in pursuing health and rehabilitation (e.g., speech-language pathology, physical and 
occupational therapy) who need and want experience with tomorrow's technology for aiding future clients. Public universities are in a prime position to guide and inspire this growing popula-
In these times of uncertain federal funding, junior and senior research faculty must begin to look elsewhere for research support. Especially in the sciences, the gold standard (particularly for pro-

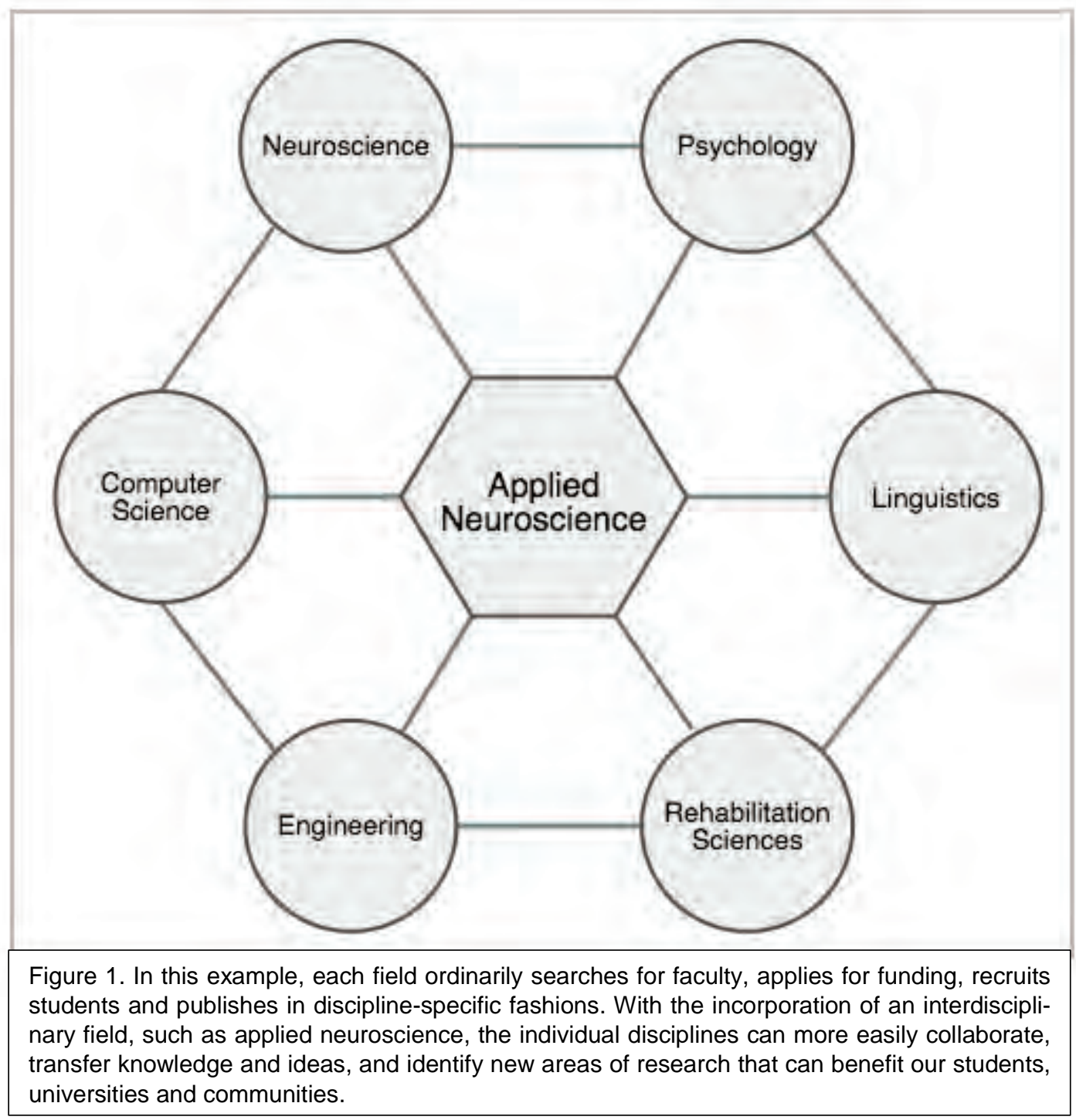

tion of young people with sophisticated skills and knowledge to pursue public collegiate education and make innovative and lasting contributions to our regions and country.

Adapting education for faculty to maximize funding. motion and tenure) has been significant external funding from the federal government (e.g., National Institutes of Health, National Science Foundation). The effect of either declining or unstable federal funding will hopefully result in an increase in funding from other sources in- 
cluding private foundations, research institutes and commercial partners. Institutional support should be increased to help investigators navigate the changing landscape of research funding, and identify new and creative models for maintaining our research programs. This is especially true for junior faculty who may have less experience in obtaining external funding.

One area of particular growth for brain-machine interfacing is collaboration with commercial partners. As an area of applied science, BMI for communication are intended to be used by individuals as an AAC device. Fortunately, a strong industry already exists for developing AAC devices with frameworks in place for interfacing with federal medical agencies including Medicare, Medicaid and the FDA. Increasing outreach to (new) faculty from the offices of technology transfer and business will be especially helpful for pursuing collaborative and sponsored projects from commercial partners for translational research applications. Finally, as a result of these changes in funding, departmental and college committees for promotion and tenure may need to reevaluate, or at least consider, the differences between candidates in federal-rich vs federal-sparse funding intervals. These discussions should result in feedback to candidate faculty to help establish a standard against which future plans may be made.

Recognizing interdisciplinary connections in your field; building interdisciplinary faculty.

Interdisciplinary and translational research programs have the potential to increase the relevance and impact of pub- lic universities on their communities. Additionally, many national funding agencies are now requiring some practical or translational outcome for research proposals. Therefore, pursuing interdisciplinary connections and collaborations will have a significant impact on the success and future of public universities in terms of local, regional and national support.

To build and maintain such programs, universities, colleges and departments may need to reconsider the method by which they recruit new faculty. Researchers with experience in multiple areas of study are prime candidates to lead interdisciplinary efforts and forge new connections between departments, programs and schools; however, these faculty candidates often do not fit in existing departmental models for faculty search and recruitment. In the past, departments may have limited the scope for faculty recruitment to either replace or augment an area of strength or bridge a gap in a domain specific area of work. To support interdisciplinary growth, departments may find it advantageous to look outside-the-box for potential faculty candidates with multiple interests and who are capable of increasing the diversity of perspectives, skills and research. Similarly, once hired, interdisciplinary faculty may need additional support to make the appropriate connections for obtaining courtesy and / or joint appointments with relevant collaborating departments and programs. Discussions with senior faculty mentors will be important to determine the impact of supplementary appointments, scholarly publishing in a variety of journals and sources of funding on the promotion and tenure process. 
Additionally, agreements between programs may be necessary for enabling primary mentoring of graduate and undergraduate students, standing on dissertation committees, and other university service opportunities. Finally, interdisciplinary collaborations within the univer- reach out to new audiences to highlight the importance and impact of public universities on their communities as well as adapting to the new realities of local and national funding. Investing in interdisciplinary and translational research can address many of these challenges and serve

\section{Facilitating interdisciplinary research}

\begin{tabular}{|c|c|}
\hline Recruitment strategy & $\begin{array}{l}\text { - Including recruits with non-traditional backgrounds } \\
\text { - Focus on the potential for interdisciplinary application of } \\
\text { applicant research }\end{array}$ \\
\hline $\begin{array}{l}\text { Professional development \& } \\
\text { mentoring }\end{array}$ & $\begin{array}{l}\text { - Continued training for managing broad scope of } \\
\text { interdisciplinary research } \\
\text { - Establishing expectations for promotion and tenure }\end{array}$ \\
\hline $\begin{array}{l}\text { Diversify funding } \\
\text { opportunities }\end{array}$ & $\begin{array}{l}\text { - Identifying alternative funding sources } \\
\text { - Draw from funding typically associated with each } \\
\text { collaborating discipline } \\
\text { - Look to commercial and foundation partners }\end{array}$ \\
\hline $\begin{array}{l}\text { University connections \& } \\
\text { networking }\end{array}$ & $\begin{array}{l}\text { - Establish appropriate relationships with collaborating } \\
\text { disciplines } \\
\text { - Enable cross-disciplinary student mentoring, course } \\
\text { instruction and research opportunities }\end{array}$ \\
\hline
\end{tabular}

Table 1: A summary of suggested considerations for growing university involvement in interdisciplinary research.

sity and beyond are sometimes best discovered and grown by student efforts. We can support these efforts through cross-department, cross-school workshops where faculty and students can meet and showcase their work. Such workshops can be expanded to regional meetings to establish strong ties with our neighbors to take advantage of our pooled resources.

\section{Summary}

Recent trends in national and local policy have led to some uncertainty for the future of the research missions of public universities. Rather than presenting an obstacle to future research, these trends may potentially increase diversity of scientific study and add to our ongoing research activities. Success during these uncertain times depends on our ability to to reiterate the need for public research in our culture and economy.

Many academic institutions are already engaging in interdisciplinary and translational research, and the suggestions discussed in this paper are designed to focus on these efforts as part of the discussion on the future of research at public universities. The main areas of emphasis include: (1) engaging our communities, especially new generations of students from primary school through college to stimulate an early interest in research, (2) adapting continuing and professional development for faculty to broaden their scope of research to help better demonstrate the need for public university research while searching for additional opportunities for alternative funding (e.g., 
private foundations and industry collaborations), and (3) discussing and planning for targeted interdisciplinary hires, and post-hiring support (e.g., considerations for promotion and tenure, crossuniversity affiliations, and infrastructure needs for interdisciplinary faculty).

Table 1 provides a succinct summary of suggested considerations for how to enhance university involvement in interdisciplinary research. Interdisciplinary research is not a solution in itself, and universities pursuing such initiatives should do so carefully to ensure sufficient planning, resources, and support are available to future interdisciplinary and translational researchers. If so, their addition will contribute toward a diverse institutional research portfolio capable of adapting to the changing landscapes of today and tomorrow.

\section{References}

Brumberg, J.S. \& Guenther, F.H., 2010. Development of speech prostheses: current status and recent advances. Expert Review of Medical Devices, 7(5), pp.667-79.

Brumberg, J.S. et al., 2010. Brain-computer interfaces for speech communication. Speech Communication, 52(4), pp.367-379.

Donchin, E., Spencer, K.M. \& Wijesinghe, R., 2000. The mental prosthesis: assessing the speed of a P300-based brain-computer interface. IEEE Transactions on Rehabilitation Engineering, 8(2), pp.174-179.

Friman, O. et al., 2007. Spelling with SteadyState Visual Evoked Potentials. In 2007 3rd International IEEE/EMBS Conference on
Neural Engineering. Kohala Coast, HI: IEEE, pp. 354-357.

Guenther, F.H., Ghosh, S.S. \& Tourville, J.A., 2006. Neural modeling and imaging of the cortical interactions underlying syllable production. Brain and Language, 96(3), pp.280-301.

Houde, J.F. \& Nagarajan, S.S., 2011. Speech production as state feedback control. Frontiers in Human Neuroscience, 5, p.82.

Indefrey, P. \& Levelt, W.J.M., 2004. The spatial and temporal signatures of word production components. Cognition, 92(1-2), pp.101-44.

Miner, L.A., McFarland, D.J. \& Wolpaw, J.R., 1998. Answering questions with an electroencephalogram-based brain-computer interface. Archives of Physical Medicine and Rehabilitation, 79(9), pp.1029-1033.

Neuper, C. et al., 2003. Clinical application of an EEG-based brain-computer interface: a case study in a patient with severe motor impairment. Clinical Neurophysiology, 114(3), pp.399-409.

Obermaier, B., Müller, G.R. \& Pfurtscheller, G., 2003. "Virtual keyboard" controlled by spontaneous EEG activity. IEEE Transactions on Neural Systems and Rehabilitation Engineering, 11(4), pp.422-6.

Plum, F. \& Posner, J.B., 1972. The diagnosis of stupor and coma. Contemporary Neurology Series, 10, pp.1-286.

Price, C.J., 2012. A review and synthesis of the first 20 years of PET and fMRI studies of heard speech, spoken language and reading. NeuroImage, 62(2), pp.816-47.

Saltzman, E.L. \& Munhall, K.G., 1989. A Dynamical Approach to Gestural Patterning in Speech Production. Ecological Psychology, 1(4), pp.333-382. 